P117

ACUTE HAEMORRHAGIC OEDEMA OF INFANCY: AN IMPORTANT SPOT DIAGNOSIS

Greg Murphy*, Thomas McGrath, Claire Hanley, Darren Roche, Asad Salim, Edna Roche. Tallaght University Hospital, Dublin, Ireland

\subsection{6/archdischild-2019-epa.472}

Background Acute haemorrhagic oedema of infancy (AHOI) or Finkelstein/Seidlmayer disease, is an uncommon leukocytoclastic small-vessel vasculitis typically occurring between the ages of 6 months and 2 years. Despite its alarming presentation, it is benign and often has a self-limiting course.

Case report A 4-year-old girl presented to the Emergency Department with a 2-day history of a diffuse spreading erythematous rash, joint pain and swelling. She was unwell for the previous 2 weeks with cough and upper respiratory tract symptoms, and was treated with a course of co-amoxiclav. Otherwise she had no relevant past medical history.

There was a widespread, macular rash that spread to form large confluent areas over the face, buttocks, torso, posterior legs, and arms [PICTURED]. The lesions had an erythematous margin with a central area of clearing. Characteristically these lesions faded in places as bruises with blue discolouration, particularly in the popliteal fossa and at the ankle, concerning for vasculitis. There was pain and swelling of both ankle joints rendering her unable to mobilise. There was a history of Sjogren's disease in the maternal grandmother.

Investigations revealed an elevated white cell count of 20.5, predominantly neutrophilic, and a C-Reactive Protein of 51. Urinalysis and blood pressure were normal. Initial treatment with prednisolone and antihistamines in ED had minimal effect. Following medical and dermatology consultation, a diagnosis of Acute Haemorrhagic Oedema of Infancy was considered.

She improved significantly over the next 48 hours without further intervention and was discharged home. There was complete resolution of her symptoms on follow up 2 days later.

Discussion AHOI is a rare, benign form of vasculitis. It typically follows a recent respiratory prodrome. There are overlapping features with Hennoch-Scönlein purpura (HSP) that have been described. Some have contemplated whether AHOI is merely a milder variant of HSP or a separate entity. Nevertheless, its dramatic presentation often results in consideration of more sinister ailments.

Skin biopsy can confirm AHOI but if identified early, the diagnosis can be made on clinical findings alone. Conservative management is the most commonly followed approach. Renal involvement and gastrointestinal bleeding are uncommon complications. Prompt recognition of this condition can spare children from invasive investigations and unnecessary treatment, therefore heightened physician awareness of this phenomenon is paramount.

\section{P118 RUSSIA'S FIRST EXPERIENCE OF POEMS AS A CHILDHOOD}

Michael Korolev*, Leonid Fedotov, Alexander Ogloblin, Alexander Kopyakov, Dmitry Baranov. St. Petersburg state pediatric medical University, Saint-Petersburg, Russian Federation

10.1136/archdischild-2019-epa.473
Introduction Achalasia of the cardia (AC) is a congenital or acquired disorder of the organ's motility, manifested by a violation of the passage of food into the stomach as a result of insufficient reflex opening of the lower sphincter of the esophagus during swallowing and disorderly peristalsis of the overlying parts of the esophageal tube.

It is $1 \%$ of all diseases of the esophagus in children, mainly in children over 3 years.

Materials and methods Patients a girl of 16 years, a boy of 11 years.

The girl from 2015, there were complaints of heaviness behind the sternum after eating, periodic vomiting, low body weight $(43 \mathrm{~kg})$. After the examination, on radiographs - sprucedale extension of the esophagus to $4 \mathrm{~cm}$, on endoscopy kardiya impassable for unit d $9 \mathrm{~mm}$, and signs of catarrhal esophagitis. Signs of AC are revealed. The clinical diagnosis was established: AC, 2 stages, dysphagia of 2-3 degrees.

Received treatment during the year nitrates, calcium channel blockers, manual therapy; no effect.

Ate only liquid food Vomiting was noted periodically. Without extraesophageal symptoms.

16.12.2016 Surgery: (POEM).

After surgery, the complication was carboxyperitoneum. Laparocentesis. On day 14, free intake of liquid food, dysphagia $0-1$ points.

On the radiograph-the appearance of a gas bubble in the stomach, the free passage of contrast throughout.

At control examination in 1 year - dysphagia 0 points. Gaining weight $(48 \mathrm{~kg})$. X-ray: the lumen of the esophagus narrowed to $2.5 \mathrm{~cm}$. The contrast flows freely into the stomach. Signs of GERD is not.

The boy in February 2015 with the acute gastroenterocolitis was hospitalized, AC was suspected by endoscopy. In may 2015, he was examined: EGDS, radiography of the esophagus with barium - revealed signs of AC of the 3rd degree, with a significant expansion of the esophagus. Dysphagia 1 point. Low body weight $-26 \mathrm{~kg}$.

In September 2017, he was hospitalized with acute pneumonia.

04.10.2017 made balloon cardiopatici. He ate adapted mixtures and wiped food. Within 14 days improvement. But complaints about the feeling of heaviness behind the sternum were protected.

26.10.2017 performed surgery - POEM.

30.10.2017 radiography- without complications.

No dysphagia.

At control examination in 1 year - dysphagia 0 points. Gaining weight $-30 \mathrm{~kg}$. Signs of GERD is not.

Summary POEM as a fairly safe and effective method of treatment of $\mathrm{AC}$, is an option of choice in children over the age of 11 years, but needs further study in children in different groups.

\section{P119 WOLF HIRSCHHORN SYNDROM (A CASE REPORT)}

${ }^{1}$ Manel Charfi, ${ }^{1}$ Chiraz Regaieg*, ${ }^{1}$ Amel Ben Hamed, ${ }^{2}$ kklas Ben Ayed, ${ }^{1}$ Amel Ben Hmed, ${ }^{1}$ Amira Bouraoui, ${ }^{1}$ Nedia Hmida, ${ }^{2}$ Hassen Kamoun, ${ }^{1}$ Afef Ben Thabet, ${ }^{1}$ Abdellatif Gargouri. ${ }^{1}$ Department of neonatology, Hedi Chaker Hospital, Sfax, Tunisia; ${ }^{2}$ Medical Genetic Department, Hedi Chaker Hospital, Sfax, Tunisia

\subsection{6/archdischild-2019-epa.474}

Introduction Wolf-Hirschhorn syndrome is a rare condition including a characteristic facial appearance, delayed growth 
and development, hypotonia, intellectual disability and seizures. Its frequency is estimated at $1 / 20000$ to $1 / 50000$ births with a female predilection. It's due to a genetic material deletion near the short arm of chromosome 4 (4p-). Through this case report we aim to remind the mains and associated features of this rare disorder as well as its management.

Methods We report the case of male newborn with a WolfHirschhorn syndrome.

Results A full term newborn was born by c-section indicated for a severe growth restriction. Birth weight was $1850 \mathrm{~g}$, length was $45 \mathrm{~cm}$ and head circumference was $30,5 \mathrm{~cm}$. Clinical examination at birth noted an important hypotonia, cyanosis with no associated signs of respiratory distress, distinctive facial features including a broad and flat nasal bridge, a high forehead, widely spaced eyes with iridian coloboma, poorly provided eyebrows, poorly formed ears with a pre-tragic fistula, micrognathia, thin lips and posterior cleft palate associated to a spina bifida occulta, posterior hypospadias, testicular ectopia and mottled skin. Echocardiography showed a type A interruption of the aortic arch. The ultrasound examination of brain and abdomen was normal. The genetic tests concluded to a wolf-Hirschhorn syndrome with a $(4 \mathrm{p})$ deletion. The boy died at the age of one month due to a severe bronchiolitis. Parental genetic tests are planned.

Conclusion Wolf-Hirschhorn is a rare genetic disorder. It may be inherited but generally it occurs as a random event. Its outcome depends on associated malformations especially congenital heart disease. We insist on the importance of genetic counseling and antenatal diagnosis when index cases are registered.

\section{P120 HYPERTENSION AND ACCESSORY RENAL ARTERIES}

${ }^{1}$ Iulia Maria Marina*, 'Orsolya Racz, ${ }^{1}$ Diana Maniu, ${ }^{2}$ Ioana Filimon, ${ }^{2}$ Otilia Fufezan, ${ }^{1}$ Simona Sorana Cainap. ' 2 nd Paediatric Clinic, University of Medicine and Pharmacy 'Iuliu Hatieganu', Cluj-Napoca, Romania; ${ }^{2}$ Radiology Department, Emergency Clinic Hospital for Children, Cluj-Napoca, Romania

\subsection{6/archdischild-2019-epa.475}

Introduction Arterial hypertension is a major risk factor for cardiovascular, cerebrovascular and renal morbidity and mortality. Renovascular disease is the third most common cause of hypertension in children after coarctation of the aorta and parenchymal renal disease. The spectrum of renovascular diseases in children is very broad compared to adults. Besides fibromuscular dysplasia and renal artery stenosis as the main causes, narrow/hypoplastic accessory renal artery or multiple accessory renal artery or multiple renal arteries could also be involved in ischemic nephropathy.

Case report We describe the case of a 15 -year-old girl who presented with palpitations, chest pain and headache. The physical examination revealed a high blood pressure (over the 99th percentile for age, gender and height). She had insignificant personal and family medical history regarding the cardiovascular risk factors. Laboratory analysis and relevant radiological evaluation were obtained in order to determine the etiology for suspected secondary hypertension. Direct plasmatic renin, plasmatic aldosterone, urinary cathecolamines, plasmatic metanephrines, vanilmandelic acid, thyroid function tests were all within normal limits. In order to rule out possible renal artery stenosis, CT angiography of the renal artery was performed, surprisingly demonstrating a small caliber (hypoplastic) accessory right superior renal artery.
Accessory renal artery stenosis/hypoplastic is a possible etiology for secondary hypertension, presumptively leading to renin secretion by the underperfused kidney.

Conclusion Physicians should be alert to the possibility of the causal role of accessory renal arteries in patients with severe and difficult to manage hypertension. For several reasons this possibility may be greater in children.

\section{\begin{tabular}{l|l} 
P121 TO SCAN OR NOT TO SCAN ...AGAIN
\end{tabular}}

Ross Foley ${ }^{*}$, Liam Skinner, Aoife Carroll. University Hospital Waterford, Waterford, Ireland

\subsection{6/archdischild-2019-epa.476}

A 13 year old male was referred to our regional centre for review of a left sided periorbital cellulitis by ENT, ophthalmology and paediatric teams. Left eye pain began 4 days previously and was initially felt to be secondary to allergies. However, one day prior to transfer to our unit intravenous cefotaxime was commenced at referring hospital and neuroimaging (CT Brain) was performed; this revealed a preseptal periorbital cellulitis with no intracranial extension. Upon arrival he was unwell and lethargic and still spiking temperatures whereupon his antimicrobial cover was broadened to include iv flucloxacillin and metronidazole.

Inflammatory markers were elevated with a CRP of 227 $\mathrm{mg} / \mathrm{L}(0-5)$ while white cell count was slightly elevated at $13 \times 10^{9} / \mathrm{L}$ (4-10). Initial blood cultures were negative but repeat blood cultures taken after 3 days of antibiotics were positive with a pathogenic gram negative organism. Following discussion with microbiology colleagues antibiotic coverage was broadened with an initial improvement over 24 hours but subsequently fevers recurred with increasing lethargy but no focal neurological signs. Inflammatory markers were slowly falling. In view of the clinical deterioration repeat imaging was requested, despite a previously normal CT Brain. MRI revealed a subdural abscess anterior to the left frontal lobe and extensive left sided sinusitis. He was emergently transferred for neurosurgical review and urgent surgery to drain the abscess and nasal sinuses. He recovered well following 6 weeks of intravenous antibiotics.

Subdural abscess is a rare but recognised complication of periorbital cellulitis, particularly secondary to sinusitis. A systematic review of the intracranial complications of rhinosinusitis revealed that the vast majority $(70 \%)$ occurred in young adolescent males with a morbidity rate of $27 \%$ and a mortality rate of $3 \%$. In our case repeat neuroimaging was undertaken due to a clinical deterioration and allowed for timely surgical intervention with a good outcome.

\section{P122 CELIAC DISEASE ASSOCIATED WITH DEPRESSION IN ADOLESCENTS}

'Orsolya-Adrienn Rácz* ${ }^{1}$ Georgia Tita, ${ }^{1}$ Alexandru Pârvan, ${ }^{2}$ Elena Predescu, ${ }^{1}$ Simona Căinap. '2nd Pediatric Clinic, University of Medicine and Pharmacy luliu Hatieganu, Cluj-Napoca, Romania; ${ }^{2}$ Child and Adolescent Psychiatry and Addiction Clinic, University of Medicine and Pharmacy luliu Hatieganu, Cluj-Napoca, Romania

\subsection{6/archdischild-2019-epa.477}

Introduction Celiac disease is an immune-mediated systemic disorder elicited by gluten which causes malabsorption and small-bowel mucosal inflammation in genetically susceptible 